\title{
On ion gyro-harmonic structuring in the stimulated electromagnetic emission spectrum during second electron gyro-harmonic heating
}

\author{
A. Samimi ${ }^{1}$, W. A. Scales ${ }^{1}$, P. A. Bernhardt ${ }^{2}$, S. J. Briczinski ${ }^{2}$, C. A. Selcher ${ }^{3}$, and M. J. McCarrick ${ }^{4}$ \\ ${ }^{1}$ The Bradley Department of Electrical and Computer Engineering, Virginia Tech, Blacksburg, VA 24061, USA \\ ${ }^{2}$ Plasma Physics Division, Naval Research Laboratory, Washington D.C., USA \\ ${ }^{3}$ Air Force Research Lab, Kirtland, NM 86117, USA \\ ${ }^{4}$ Marsh Creek, LLC, Gakona, Alaska, USA \\ Correspondence to: A. Samimi (arsamimi@vt.edu)
}

Received: 9 March 2012 - Revised: 27 October 2012 - Accepted: 8 November 2012 - Published: 26 November 2012

\begin{abstract}
Recent observations show that, during ionospheric heating experiments at frequencies near the second electron gyro-harmonic, discrete spectral lines separated by harmonics of the ion-gyro frequency appear in the stimulated electromagnetic emission (SEE) spectrum within $1 \mathrm{kHz}$ of the pump frequency. In addition to the ion gyro-harmonic structures, on occasion, a broadband downshifted emission is observed simultaneously with these spectral lines. Parametric decay of the pump field into upper hybrid/electron Bernstein (UH/EB) and low-frequency ion Bernstein (IB) and oblique ion acoustic (IA) modes is considered responsible for generation of these spectral features. Guided by predictions of an analytical model, a two-dimensional particle-in-cell (PIC) computational model is employed to study the nonlinear processes during such heating experiments. The critical parameters that affect the spectrum, such as whether discrete gyroharmonic on broadband structures is observed, include angle of the pump field relative to the background magnetic field, pump field strength, and proximity of the pump frequency to the gyro-harmonic. Significant electron heating along the magnetic field is observed in the parameter regimes considered.
\end{abstract}

Keywords. Ionosphere (Active experiments; Plasma waves and instabilities)

\section{Introduction}

Strong, high-frequency electromagnetic (EM) waves transmitted into the ionosphere during ionospheric heating experiments generate secondary electrostatic (ES) and electro- magnetic waves through nonlinear plasma processes. The power spectrum of the signal acquired by ground-based receivers shows these new generated waves, so-called stimulated electromagnetic emission (SEE), that are frequency shifted within $100 \mathrm{kHz}$ relative to the heater or pump frequency (Thidé et al., 1982).

Understanding of this process is critical from both practical and theoretical standpoints. Various SEE spectral features can be studied as diagnostic tools that give information about the condition of the ionosphere and nonlinear plasma processes (Leyser, 2001). For instance, electron temperature (Bernhardt et al., 2009) or amplitude of the local geomagnetic field (Leyser, 1992) in the interaction region may be estimated. Parametric decay of the pump field into new plasma waves has been introduced as a fundamental process that generates SEE. The EM pump wave can be directly involved in the decay process and generate new EM and ES waves. For example, decay of the pump field into ion acoustic or electrostatic ion cyclotron waves and scattered EM wave is involved in the stimulated Brillouin scatter process (Norin et al., 2009; Bernhardt et al., 2009). On the other hand, for other SEE features, the EM pump undergoes conversion to another ES wave which then decays into new ES waves that are then back converted to EM waves. For example, the downshifted maximum (DM) spectral feature is proposed to be generated by this process (Zhou et al., 1994; Bernhardt et al., 1994).

Recent experimental observations of SEE during second electron gyro-harmonic heating show structures ordered by harmonics of the ion gyro-frequency (Bernhardt et al., 2011). The spectrum may show up to sixteen discrete spectral lines and is distinctly different from magnetized stimulated 

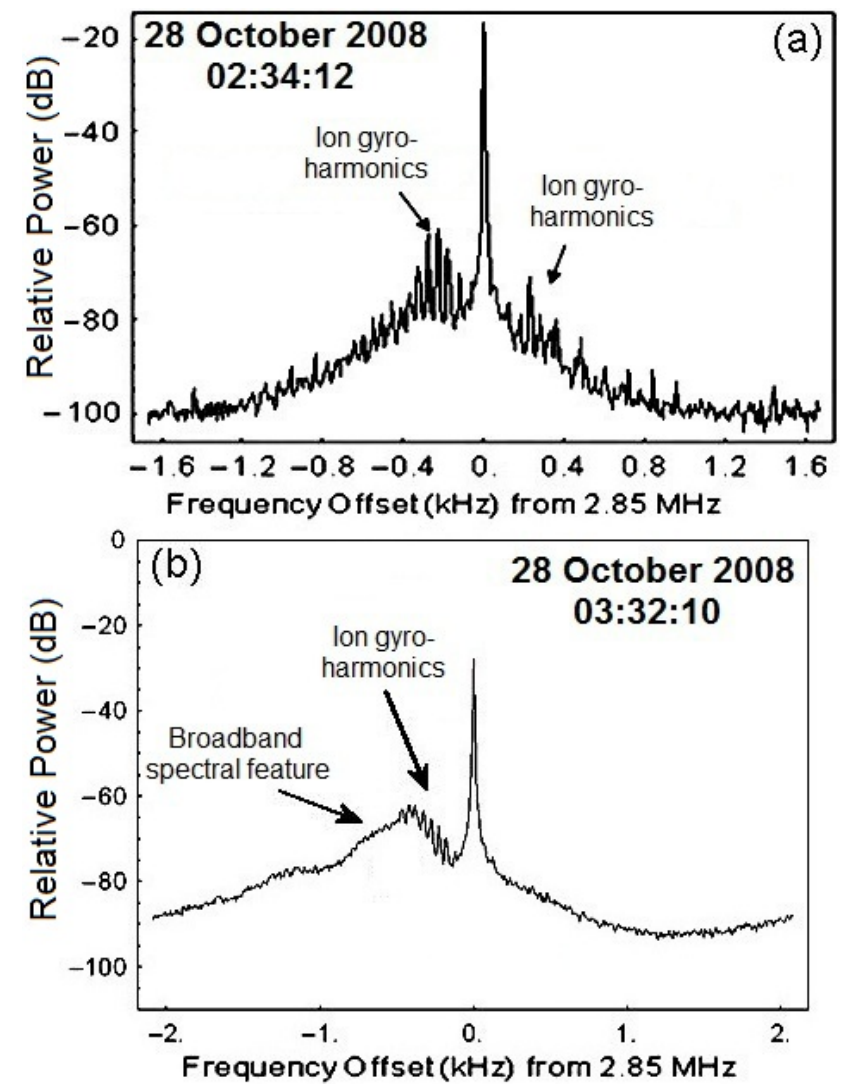

Fig. 1. Experimental observations of (a) ion gyro-harmonic structures and (b) simultaneous broadband and ion gyro-structures observed at HAARP during which heater frequency was tuned close to second electron gyro-harmonic frequency.

Brillouin scatter involving decay into electrostatic ion cyclotron waves in which only one spectral line downshifted by ion gyro-frequency is observed (Bernhardt et al., 2009). Brillouin scatter is an electromagnetic parametric process of direct conversion of the EM wave into the high-frequency EM sideband and low-frequency ES wave in the long wavelength regime $\left(k_{\perp} \rho_{\mathrm{i}}<1\right.$ where $k_{\perp}$ is the perpendicular wavenumber and $\rho_{\mathrm{i}}$ is the ion gyro-radius). The process here, as will be discussed, involves conversion of the EM wave first into an ES upper hybrid/electron Bernstein (UH/EB) wave, then an electrostatic parametric process (short wavelength regime $k_{\perp} \rho_{\mathrm{i}}>1$ ), and finally backscattering into the EM wave observed as SEE. Cascading during Brillouin scatter has been observed to produce additional lines (one or maximum two) but not a large number as observed in these experiments. Also, the amplitude of these lines greatly decreases with harmonic number with offset from the pump frequency unlike the observations to be described here. It should be noted that our very recent experiments show that magnetized Brillouin scatter is clearly suppressed for the pump frequency near the second gyro-harmonic (also third) while these gyro-features are enhanced, again consistent with theory. A more careful comparison of these two processes is beyond the scope of the current manuscript, and this is the subject of ongoing work that will be presented in a future publication.

The original explanation for the gyro-harmonic structuring was parametric decay of the pump EM wave into electron and pure ion Bernstein waves $\left(k_{\|}=0\right.$ where $k_{\|}$is the wavenumber along the geomagnetic field) (Bernhardt et al., 2011). In addition to the ion gyro-harmonic structures, some of the experimental measurements also exhibit a broadband spectral feature downshifted from the pump frequency within $1 \mathrm{kHz}$ that coexists with the ion gyro-harmonic structures. The object of this paper is to investigate the generation of ion gyroharmonic and associated broadband spectral features within $1 \mathrm{kHz}$ of the pump frequency and to provide fundamental parameters that characterize these types of spectra that may have critical diagnostic information of the heated plasma. Also for the first time, a computational model will be used to follow the nonlinear evolution more accurately than has been possible in the past. This paper is organized as follows. In the next section, experimental observations are provided. An analytical model is then used to provide guidance on important parameters that characterize the proposed parametric instability process. Section 4 is devoted to the computational model and results. Finally, conclusions are provided.

\section{Experimental observations}

In order to obtain the SEE spectra, a $30 \mathrm{~m}$ folded dipole antenna with a receiver with around $90 \mathrm{~dB}$ dynamic range was set up by the Naval Research Laboratory (NRL) close to High Frequency Active Auroral Research Program (HAARP) site (63.09 ${ }^{\circ} \mathrm{N}$ Lat., $-145.15^{\circ} \mathrm{E}$ Long.) in Gakona, Alaska. The receiver shifts the frequency of the acquired signal by the heater frequency by mixing, and sampling it at $250 \mathrm{kHz}$. The acquired data are post-processed by utilizing the fast Fourier transform (FFT) to obtain spectrograms of the received signal. During the experiment the pump duty cycle was $4 \mathrm{~min}$ on/4 min off and heater frequency was set to $2.85 \mathrm{MHz}$. This is almost twice the electron gyro-frequency above HAARP, calculated using the International Geomagnetic Reference Field (IGRF) model. The digital ionosonde at HAARP estimated the reflection altitude was $221 \mathrm{~km}$. At $221 \mathrm{~km}$ altitude, the magnetic field strength is $B=0.052632 \mu T$, the electron gyro-frequency $\Omega_{\mathrm{ce}}=e B / m_{\mathrm{e}}=2 \pi\left(1.47 \times 10^{6}\right) \mathrm{rad} \mathrm{s}^{-1}$, and the ion gyro-frequency is $\Omega_{\mathrm{ci}}=e B / m_{\mathrm{i}}=2 \pi(50.1) \mathrm{rad} \mathrm{s}^{-1}$ where $m_{\mathrm{e}}$ and $m_{\mathrm{i}}$ are the electron and ion masses. The experiment was carried out in O-mode during which the heater was operating at full power, i.e. $3.6 \mathrm{MW}$, and effective radiated power is estimated to be $280 \mathrm{MW}$. The heater beam was pointed to magnetic zenith with an azimuth of 202 degrees and a zenith angle of 14 degrees.

Figure 1 shows the power spectrum of the acquired signal for two experiments with almost $1 \mathrm{~h}$ difference between measurements. In the first experiment, conducted at 02:34 UT 
on 28 October 2008 (around the local sunset), distinct spectral structures at harmonics of ion gyro-frequency are evident in the upshifted and downshifted sidebands where in the downshifted side 16 spectral lines are distinguishable and the strongest is the fourth harmonic. Harmonic interference of the power supply at multiples of $120 \mathrm{~Hz}$ is also seen in the spectrum and should not be mistaken as the SEE feature. In the second measurement that was carried out at 03:32 UT, in addition to ion gyro-harmonic structures, a broadband spectral feature that is peaked at around $500 \mathrm{~Hz}$ below the pump field frequency appears in the spectra. Similar characteristics were repeatable during other observations. Experimental observations of the discrete spectral structures were reported in Bernhardt et al. (2011). However, the broadband feature and its relation to the discrete structures is presented in this paper for the first time.

\section{Analytical model}

Parametric decay of the pump field into upper hybrid/electron Bernstein and neutralized ion Bernstein (IB) waves (with important differences in dispersive characteristics from pure ion Bernstein waves) has been proposed as a viable process for generation of these spectral features (Scales et al., 2011). While pure IB waves propagate perpendicular to the background magnetic field, neutralized IB waves propagate slightly off-perpendicular, i.e. $k_{\|} / k_{\perp} \geq$ $\sqrt{m_{\mathrm{e}} / m_{\mathrm{i}}}$. These waves exhibit neutralizing Boltzmann electron behavior and have closer dispersive relationships and phenomenological behavior to ion acoustic waves. By enforcing wavenumber and frequency matching conditions, i.e. $\boldsymbol{k}_{0}=\boldsymbol{k}_{1}+\boldsymbol{k}_{\mathrm{s}}$ and $\omega_{0}=\omega_{1}+\omega_{\mathrm{s}}$, where subscripts 0,1 and $s$ represent parameters of the pump field, the high-frequency decay mode and the low-frequency decay mode respectively, the general dispersion relation describing weak coupling is (Porkolab, 1974)

$\varepsilon\left(\omega_{\mathrm{s}}\right)+\frac{\beta_{\mathrm{e}}^{2}}{4} \chi_{\mathrm{i}}\left(\omega_{\mathrm{s}}\right)\left\{\frac{\varepsilon_{\mathrm{e}}\left(\omega_{\mathrm{s}}\right)}{\epsilon_{\mathrm{e}}\left(-\omega_{1}^{*}\right)}-2\right\}=0$

where $\varepsilon(\omega)=1+\chi_{\mathrm{e}}(\omega)+\chi_{\mathrm{i}}(\omega)$, and $\varepsilon_{\mathrm{e}}(\omega)=1+\chi_{\mathrm{e}}(\omega)$. The susceptibility of the $j$-th species is given by

$\chi_{j}(\omega)=\frac{1}{k^{2} \lambda_{D j}^{2}}\left\{\frac{1+\zeta_{j 0} \sum_{n=-\infty}^{\infty} \Gamma_{n}\left(b_{j}\right) Z\left(\zeta_{j n}\right)}{1+\frac{i v_{j}}{k_{\|} v_{t j}} \sum_{n=-\infty}^{\infty} \Gamma_{n}\left(b_{j}\right) Z\left(\zeta_{j n}\right)}\right\}$

where $b_{j}=k_{\perp}^{2} \rho_{j}^{2}, k$ is the wavenumber, $k_{\perp}\left(k_{\|}\right)$is the component of $k$ perpendicular (parallel) to the magnetic field $\boldsymbol{B}$, $\rho_{j}$ is the gyro-radius, $\zeta_{j n}=\left(\omega+i v_{j}-n \Omega_{n}\right) / k_{\|} v_{t j}, \Omega_{n}$ is the gyro-frequency, $v_{t j}$ is the thermal velocity, $v_{j}$ is the collision frequency, $\Gamma_{n}\left(b_{j}\right)=I_{n}\left(b_{j}\right) \exp \left(-b_{j}\right), Z$ is the Fried-Conte function, $I_{n}$ is the modified Bessel function of the first kind of order $n, \lambda_{D j}$ is the Debye length. $\beta_{\mathrm{e}}$, the coupling coefficient, proportional to the pump field $E_{0}$, is given by

$$
\begin{aligned}
\beta_{\mathrm{e}}=\frac{e}{m_{\mathrm{e}}} & \left\{\left(\frac{E_{0 \|} k_{\|}}{\omega_{0}^{2}}+\frac{E_{0 x} k_{x}+E_{0 y} k_{y}}{\omega_{0}^{2}-\Omega_{\mathrm{ci}}^{2}}\right)^{2}\right. \\
& \left.+\frac{\left(E_{0 x} k_{y}-E_{0 y} k_{x}\right)^{2} \Omega_{\mathrm{ce}}^{2}}{\omega_{0}^{2}\left(\omega_{0}^{2}-\Omega_{\mathrm{ci}}^{2}\right)^{2}}\right\}^{1 / 2}
\end{aligned}
$$

and $k_{\perp}^{2}=k_{x}^{2}+k_{y}^{2}$. For simplicity, collisional effects will be neglected when discussing solutions of Eq. (2). It is assumed the pump field strength is above the threshold for the instability and emphasis is placed in part on considering the structure of the predicted spectrum.

The pump wave is modeled using the dipole approximation, i.e. $k_{0}=0$. A more refined approach would be to consider the pump wavenumber to be given by the wavenumber of the irregularities generated by the oscillating two stream instability OTSI (Huang and Kuo, 1995). The simplified approximate approach here is deemed adequate for initial interpretations of the experimental data. Future work will consider more refined calculations.

The Bernstein modes propagate almost perpendicular to the magnetic field. Therefore, it is assumed the parametric decay process occurs at the upper hybrid altitude in which the electric field is almost perpendicular to the geomagnetic field (Leyser, 1991); also the double resonance condition is assumed for second gyro-harmonic heating, i.e. $\omega_{0} \approx$ $\omega_{\mathrm{uh}}=2 \Omega_{\mathrm{ce}}$. Although this model is valid for weak coupling $\left(\beta_{\mathrm{e}}<1\right)$, it still provides guidance for the more general computational model of the following section. The full dispersion relation is solved numerically for various parameter regimes. Two cases are discussed here in which oxygen ions are assumed, the pump field frequency $\omega_{0}=2 \Omega_{\mathrm{ce}}-40 \Omega_{\mathrm{ci}}$ and the electron to ion temperature ratio $T_{\mathrm{e}} / T i=3$ (Bernhardt et al., 2011). Furthermore, the pump field is described by the electron oscillating velocity, i.e. $v_{\mathrm{osc}}=e E_{0} / m_{\mathrm{e}} \omega_{0}$ where $e$ is the electron charge. In the growth rate calculations, $v_{\text {osc }} / v_{\text {the }}=0.5$ corresponding to $E_{0} \approx 10 \mathrm{~V} \mathrm{~m}^{-1}$ is used. The electric field is assumed to be slightly off-perpendicular to the background magnetic field, and the off-perpendicular angle is denoted by $\theta_{E}$. Figure 2 shows the dispersion relation of the low-frequency decay mode (shift of the destabilized wave from the pump frequency) and the corresponding growth rates (i.e. $\omega_{\mathrm{S}}=\omega_{\mathrm{r}}+j \gamma$ ) for two cases: $\theta_{E}=5.3^{\circ}$ and $\theta_{E}=7.6^{\circ}$. The left vertical axis is normalized frequency (solid curves); the right is normalized growth rate (dashed curves); and the horizontal axis is the perpendicular normalized wavenumber. It is clear that, at small off-perpendicular angles $\left(\theta_{E} \approx 5^{\circ}\right)$, a discrete band of upper hybrid/electron Bernstein (UH/EB) waves is destabilized and shifted below the pump frequency by multiples of the ion gyro-frequency. This is parametric decay of the pump field into UH/EB and neutralized IB waves (otherwise known as electrostatic ion cyclotron harmonic waves; e.g. Kindel and Kennel, 1971). 

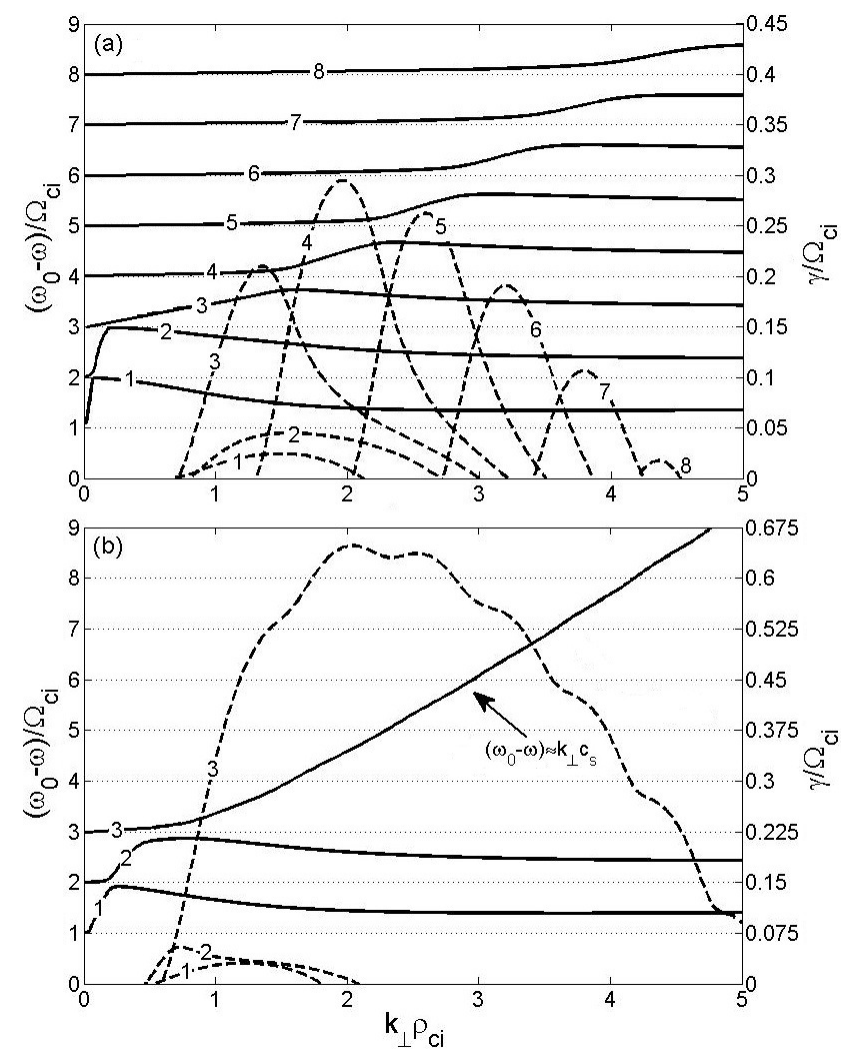

Fig. 2. Dispersion relation (solid lines) and growth rate (dashed lines) for (a) neutralized ion Bernstein decay instability for $\theta_{E}=5.3^{\circ}$ and $v_{\mathrm{osc}} / v_{\text {the }}=0.5$ and (b) neutralized ion Bernstein and oblique ion acoustic decay instabilities for $\theta_{E}=7.6^{\circ}$ and $v_{\mathrm{osc}} / v_{\text {the }}=0.5$. Note that $\rho_{\mathrm{ci}} \approx 2 \mathrm{~m}$ and $\Omega_{\mathrm{ci}} \approx 50 \mathrm{~Hz}$.

The neutralized ion Bernstein waves exist for $k_{\|} / k_{\perp} \sim 0.1$ and have dispersion relation $\omega \approx n \Omega_{\mathrm{ci}}\left[1+\frac{T_{\mathrm{e}}}{T_{\mathrm{i}}} \Gamma_{n}\left(k_{\perp}^{2} \rho_{\mathrm{i}}^{2}\right)\right]$ for the $n$-th harmonic number. For these parameters, maximum growth occurs in relatively narrow frequency bands with downshifts approximately at $\left(n+\frac{1}{2}\right) \Omega_{\mathrm{ci}}$.

At slightly higher off-perpendicular angles of the pump field, $\theta_{E} \approx 8^{\circ}$, a broadband mode is destabilized as well as lower harmonic discrete modes. This is the decay of the pump field into UH/EB and neutralized IB waves, and also highly oblique ion acoustic IA waves with dispersion relation $\omega \approx k_{\perp} c_{\mathrm{S}}$ where $c_{\mathrm{s}} \approx \sqrt{K T_{\mathrm{e}} / m_{\mathrm{i}}}$ is the sound speed and $K$ is the Boltzmann constant. $\theta_{E}$ is an important parameter in determining the transition from discrete ion Bernstein (IB) decay to broadband oblique ion acoustic (IA) decay. Equation (1) shows this may also be influenced by parameters $v_{\text {osc }} / v_{\text {the }}$ and $\left|\omega_{0}-2 \Omega_{\text {ce }}\right| / \Omega_{\text {ce }}$. We should emphasize that both of the parametric decay processes that have been discussed here occur in the upper hybrid altitude in which electric field has a specific angle relative to the magnetic field. Electron density, its gradient relative to the height in the interaction region and the direction of the transmitter beam are three critical parameters that determine the angle of the E- field relative to the magnetic field. Our calculations show that the threshold electric field intensity required to excite the oblique IA wave is actually higher than for the neutralized IB mode. Since the oblique IA wave is excited by stronger electric fields (i.e. $v_{\mathrm{osc}} / v_{\text {the }}$ ), its growth rate is larger than the neutralized IB modes. It is broadband since, for higher growth rates, the growth rate is near the ion gyro-frequency $\gamma \approx \Omega_{\mathrm{ci}}$ and the ions are unmagnetized. It can be shown in the analytical calculations that reducing the E-field intensity causes the discrete neutralized IB modes to be excited rather than the broadband IA mode as the ions become magnetized. Experimental observations also show that the broadband feature develops faster than the discrete structures.

Therefore, there are actually at least two important parameters that determine whether there is a discrete or broadband spectrum. These are the pump field strength as well as the electric field angle. From a practical point during an experiment, this transition may be prompted by varying either pump power or propagation effects (such as possible change in beam angle or ionospheric parameters that influence propagation). Further experiments are required to carefully validate this transition. On the other hand, the most important parameter determining the discrete IB decay line with maximum growth (e.g. 4 in Fig. 2 ) appears to be $\left|\omega_{0}-2 \Omega_{\text {ce }}\right| / \Omega_{\text {ce }}$. Note, in the aforementioned calculations, the electric field intensity of the pump is assumed to be $E_{0}=10 \mathrm{~V} \mathrm{~m}^{-1}$ (a round figure used for demonstration purposes), and the threshold intensity required to excite the instability is $E_{0}=3.5 \mathrm{~V} \mathrm{~m}^{-1}$.

The presumption of the discussed generation mechanism is that the pump EM wave is converted into the electrostatic pump UH/EB wave. The thermal oscillating two stream instability (OTSI) is the mechanism that has been proposed for the conversion of the EM wave into the electrostatic UH/EB pump wave (e.g. Huang and Kuo, 1994). It has been shown by these authors that the field amplitude for this OTSI driving process actually has a relatively low threshold of $1 \mathrm{~V} \mathrm{~m}^{-1}$ or less. Initial simulations, to be described in more detail in the next section, indicate that the resulting OTSI field (from the pump EM field) should then grow significantly large enough for driving the parametric decay instability. In order to check effect of the electron to ion temperature ratio on the spectral features, we did similar calculations for $T_{\mathrm{e}} / T_{\mathrm{i}}=1$. For $T_{\mathrm{e}} / T_{\mathrm{i}}=1$, just the first five modes of the neutralized IB wave are destabilized at $\theta_{E}=8.2^{\circ}$. Also, the transition boundary between exciting IB modes and IA mode shifts to $\theta_{E} \geq 10^{\circ}$ in this case.

\section{Simulation model and results}

In order to study nonlinear processes involved in generation of ion gyro-harmonic structures more thoroughly, a periodic two space and three velocity dimension (2D3V) magnetized electrostatic particle-in-cell (PIC) (Birdsall and Langdon, 1991) model is used. It is assumed that the background 
magnetic field is along the $\mathrm{z}$-axis. The long wavelength pump wave is modeled by the dipole approximation (i.e. $\boldsymbol{E}=$ $\left.\left[E_{0} \sin \left(\theta_{E}\right) \hat{z}+E_{0} \cos \left(\theta_{E}\right) \hat{y}\right] \cos \left(\omega_{0} t\right)\right)$. The length along $B$ is $256 \lambda_{D}$ and its length perpendicular to $B$ is $512 \lambda_{D}$, and 50 particles per cell per species are used for sufficiently low noise level. The initial velocity distributions are Maxwellian for both electrons and ions and $T_{\mathrm{e}}=T_{\mathrm{i}}$. The ion to electron mass ratio $m_{\mathrm{i}} / m_{\mathrm{e}}=200$ is sufficient for separating electron and ion timescales and provides reasonable computational efficiency. For scaling purposes, $\lambda_{D} \sim 1 \mathrm{~cm}$ and $v_{\text {the }} \sim$ $2 \times 10^{5} \mathrm{~m} \mathrm{~s}^{-1}$ at altitudes of interest.

A number of simulations were conducted for a variety of parameter regimes. Two cases are discussed here for which the pump field is applied during the whole simulation period $v_{\text {osc }} / v_{\text {the }}=2.8$, reasonably consistent with its estimated strength in the interaction region (Bernhardt et al., 2009). The pump frequency is set slightly above $2 \Omega_{\text {ce }}$, i.e. $\omega_{0}=2 \Omega_{\text {ce }}+$ $0.1 \Omega_{\text {ci }}$ (note that $\left|\omega_{0}-2 \Omega_{\text {ce }}\right| / \Omega_{\text {ce }}$ is comparable for both analytical and simulation models). In the first case, $\theta_{E}=18^{\circ}$ and in the second, $\theta_{E}=24^{\circ}$. Figure 3 shows the time evolution of the electrostatic field energy $\left(W_{E}=\frac{1}{2} \epsilon_{0} \int|E|^{2} d v\right.$ in relative simulation units). Initially in the $E_{\mathrm{y}}$ energy, the lower hybrid (LH) parametric decay instability (also predicted by Eq. (1) but growth rate calculation not shown) is observed associated with the prominent downshifted maximum (DM) SEE spectral feature. Afterwards, in the $E_{\mathrm{z}}$ field energy, the ion Bernstein parametric decay instability can be seen to develop. Note that the overshoot in the $E_{\mathrm{y}}$ energy is related to the second phase of the growth of the LH parametric decay instability. The energy first increases, then reaches a quasiequilibrium state and then again starts to increase until it reaches a saturation state before decaying. This is indicative of the pump being continuously applied. It should be noted that frequency power spectra during the LH parametric decay phase in the case of both angles show sidebands upshifted and downshifted from the pump frequency by the lower hybrid frequency (not shown). The growth rate from the field energy during the IB/IA parametric decay phase is comparable in both angle cases $\left(\gamma / \Omega_{\mathrm{ci}} \sim 0.1\right)$, but the $\theta_{E}=24^{\circ}$ case is slightly larger as qualitatively predicted from Eq. (1).

To consider the power spectrum for comparison with observations, the current density frequency spectrum along the magnetic field (z-direction) at a fixed point (i.e. $\left|J_{\mathrm{Z}}(\omega)\right|^{2}$ ) is shown in Fig. 4 for $\theta_{E}=18^{\circ}$ and $\theta_{E}=24^{\circ}$. Similar to the analytical model predictions, at smaller off-perpendicular angles $\left(\theta_{E}=18^{\circ}\right)$ the power spectrum of $\left|J_{Z}(\omega)\right|^{2}$ exhibits discrete structures shifted below the pump frequency by harmonics of the ion-gyro frequency and at higher offperpendicular angles $\left(\theta_{E}=24^{\circ}\right)$ a broadband spectral feature appears and coexists with lower gyro-harmonics near the pump frequency. The frequency spectrum of the current density across the magnetic field (i.e. $\left|J_{\mathrm{y}}(\omega)\right|^{2}$ ) exhibits sidebands shifted below and above the pump frequency by $\omega_{\mathrm{LH}}$ due to the lower hybrid parametric decay instability (Hussein and Scales, 1997). This is believed to produce the down-
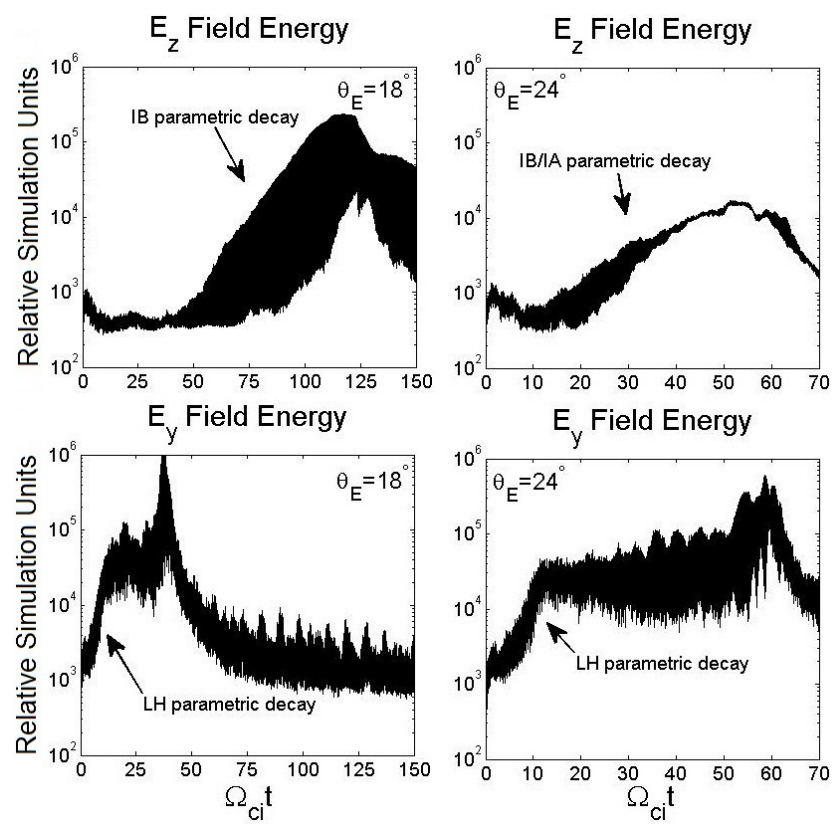

Fig. 3. Simulated time evolution of electrostatic field energy parallel (z-field) and perpendicular (y-field) to the background magnetic field shows development of lower hybrid (LH) decay instability (y-field) for both cases, IB decay instability (z-field) for the case $\theta_{E}=18^{\circ}$ and neutralized IB/oblique IA decay instabilities (z-field) for the case $\theta_{E}=24^{\circ}$.

shifted maximum and upshifted maximum emission lines in the SEE spectrum (Leyser, 2001). On these timescales the amplitude of the current density along and across the magnetic field depends upon the growth and the decay of the LH and the IB parametric decay instability. Thus, the current density is not necessarily the strongest along the pump field direction.

Figure 5 shows the time evolution of the electron kinetic energy, $K_{\mathrm{e} \|}=\frac{1}{2} \sum m_{\mathrm{e}} v_{\mathrm{e} \|}^{2}$, and also the velocity distribution function both along the magnetic field at the end of the simulation. There is significant heating along the magnetic field due to the development of the parametric instabilities. The heating is result of the collisionless damping (i.e. waveparticle heating). For these parameters, there is more heating for $\theta_{E}=18^{\circ}$ which indicates more local heating associated with the gyro-harmonics relative to the broadband oblique IA structure. Less free energy can be seen to go into total electrostatic field energy in this case. Note that the free flowing electrons along the magnetic field are not considered in the current model due to employing the periodic boundary condition. This would require refreshing the particles when they leave the system along the magnetic field. This is not considered too great of a limitation due to the timescale of the simulation, which is primarily to consider basic physics of the parametric decay process. This alteration of the boundary condition will be made on a future version of the simulation model. With the current model, the electron kinetic energies 

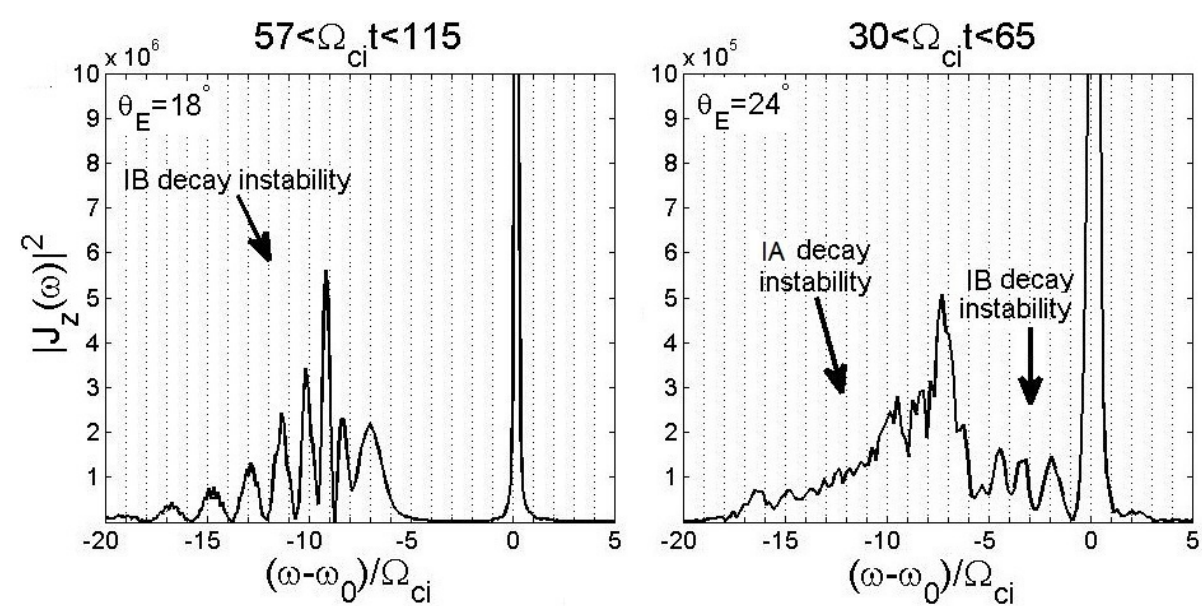

Fig. 4. Power spectrum of simulated current density along the magnetic field taken over time ranges corresponding to IB/IA parametric decay for $\theta_{E}=18^{\circ}$ shows discrete ion gyro-harmonic structures and for $\theta_{E}=24^{\circ}$ shows broadband oblique IA spectral feature.
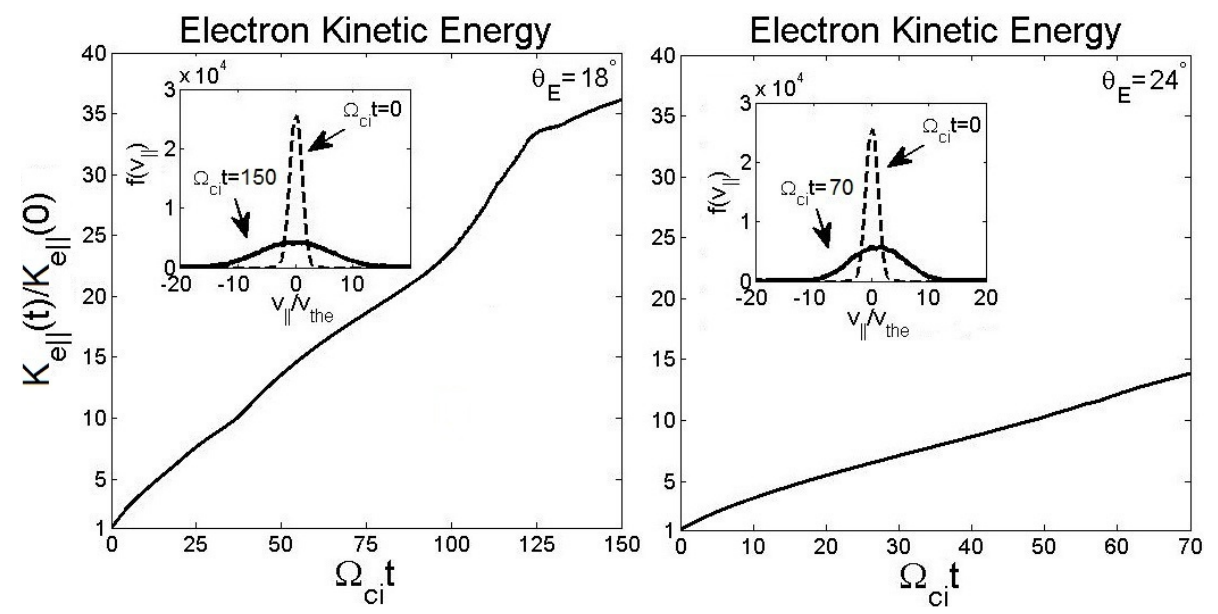

Fig. 5. Time evolution of parallel electron kinetic energy and corresponding electron velocity distribution functions at the end of the simulation for $\theta_{E}=18^{\circ}$ and $\theta_{E}=24^{\circ}$.

perpendicular to the magnetic field show much less growth in comparison to the parallel component of the kinetic energies. This behavior is relatively similar for the cases of the two angles. The significant acceleration of the electrons along the magnetic field will have connections to airglow, which is of interest for a spectrum of diagnostics in the heated volume. Correlation of the spectral features with such diagnostics will also be quite useful during future experiments.

\section{Conclusions}

In this study, parametric decay of the pump field into another $\mathrm{UH} / \mathrm{EB}$ and neutralized IB waves is proposed as a viable process for generation of ion-gyro structures in the SEE spectra. It is found that this process can occur at the upper hybrid altitude where the electric field is almost perpendicular to the geomagnetic field. Characteristics of the spectrum are pre- dicted to change from discrete harmonics to more broadband involving parametric decay into broadband oblique IA mode depending on the orientation of the pump field relative to the background geomagnetic field, $\theta_{E}$. During heating experiments, varying the heater antenna beam angle relative to the geomagnetic field is expected to effectively vary this parameter and produce important variations in the SEE spectrum. However, this is just one important parameter that influences and characterizes the SEE gyro-harmonic features and associated nonlinear plasma processes in this frequency range. Some preliminary predictions made here include the pump wave amplitude and frequency relative to the second electron gyro-harmonic. The strongest excited SEE gyro-harmonic line depends on the frequency offset of the pump field relative to the second electron gyro-harmonic. Moreover, in addition to the off-perpendicular angle of the pump field relative to the geomagnetic field, the strength of the electric field 
also determines whether the oblique IA wave or the neutralized IB modes are excited. For a fixed $\theta_{E}$ angle, for a small pump field strength, the neutralized IB modes are excited (producing the discrete SEE spectrum) while for the higher pump field intensities, the oblique IA mode is excited (producing the broadband spectrum). As $\theta_{E}$ reduces, it is more difficult to excite oblique IA modes even by using stronger pump powers.

Note that a similar generation mechanism that is proposed for the broadband feature has been introduced for the downshifted peak (DP) feature observed during the heating near the third electron gyro-harmonic (Huang and Kuo, 1995). It is possible that these two features have similar physical characteristics; however, a final conclusion will require comparison experiments at the second and third electron gyrofrequencies. Differences should be noted however. First the broadband feature here occurs near $500 \mathrm{~Hz}$. The DP is near $1.5 \mathrm{kHz}$, so there is a significant difference in frequency. Both features appear to be enhanced for the pump frequency near the electron gyro-harmonic. From a theoretical standpoint, note that the broadband structure here may have some structuring at ion gyro-harmonic frequencies due to ion cyclotron damping. The work by Huang and Kuo considered only a cold plasma ion susceptibility where the susceptibility here is fully kinetic which allows for these physical effects. Other experimental checks predicted by the theory to compare the two lines are as follows: (1) transition from discrete to broadband structure with increasing pump power and (2) transition from discrete to broadband structure with varying beam angle. In summary, due to considerable diagnostic information available from these two new SEE spectral features and other related SEE spectral features (Sharma et al., 1993; Huang and Kuo, 1995; Tereshchenko et al., 2006), a comprehensive study of the impact of various parameter regimes on the parametric instabilities and further experimental observations are being conducted and will be presented in the future.

Acknowledgements. The work at Virginia Tech is supported by the National Science Foundation. The work at the Naval Research Laboratory was supported by the NRL 6.1 base program. S. J. Briczinski is sponsored by the NRC postdoc program.

Editor-in-Chief M. Pinnock and Topical Editor P.-L. Blelly thank T. Leyser for his help in evaluating this paper.

\section{References}

Bernhardt, P. A., Wagner, L. S., Goldstein, J. A., Trakhtengerts, V. Yu., Ermakova, E. N., Rapoport, V. O., Komrakov, G. P., and Babichneko, A. M.: Enhancement of stimulated electromagnetic emission during two frequency ionospheric heating experiments, Phys. Rev. Lett., 72 2879-2882, doi:10.1103/PhysRevLett.72.2879, 1994.

Bernhardt, P. A., Selcher, C. A., Lehmberg, R. H., Rodriguez, S., Thomason, J., McCarrick, M., and Frazer, G.: Determination of the electron temperature in the modified ionosphere over HAARP using the HF pumped Stimulated Brillouin Scatter (SBS) emission lines, Ann. Geophys., 27, 4409-4427, doi:10.5194/angeo-27-4409-2009, 2009.

Bernhardt, P. A., Selcher, C., and Kowtha, S.: Electron and ion Bernstein wave excited in the ionosphere by high power EM waves at the second harmonic of the electron cyclotron frequency, Geophys. Res. Lett., 38, L19107, doi:10.1029/2011GL049390, 2011.

Birdsall, C. K. and Langdon, A. B.: Plasma physics via computer simulation, New York, 1991.

Huang, J. and Kuo, S. P.: Cyclotron harmonic effect on the thermal oscillating two-stream instability in the high latitude ionosphere, J. Geophys. Res., 99, 2173-2181, doi:10.1029/93JA02668, 1994.

Huang, J. and Kuo, S. P.: A Generation Mechanism for the Downshifted Peak in Stimulated Electromagnetic Emission Spectrum, J. Geophys. Res., 100, 21433-21438, doi:10.1029/95JA02302, 1995.

Hussein, A. A. and Scales, W. A.: Simulation studies of parametric decay processes associated with ionospheric stimulated radiation, Radio Sci., 32, 2099-2107, doi:10.1029/97RS01349, 1997.

Kindel, J. M. and Kennel, C. F.: Topside current instabilities, J. Geophys. Res., 76, 3055-3078, doi:10.1029/JA076i013p03055, 1971.

Leyser, T. B.: Parametric interaction between upper hybrid and lower hybrid waves in heating experiments, Geophys. Res. Lett., 18, 408-411, doi:10.1029/91GL00136, 1991.

Leyser, T. B., Thidé, B., Goodman, S., Waldenvik, M., Veszelei, E., Grach, S. M., Karashtin, A. N., Komrakov, G. P., and Kotik, D. S.: Narrow cyclotron harmonic absorption resonances of stimulated electromagnetic emission in the ionosphere, Phys. Rev. Lett., 68, 3299-3302, doi:10.1103/PhysRevLett.68.3299, 1992.

Leyser, T. B.: Stimulated electromagnetic emission by highfrequency electromagnetic pumping of the ionospheric plasma, Space Sci. Rev., 98, 223-328, doi:10.1023/A:1013875603938, 2001.

Norin, L., Leyser, T. B., Nordblad, E., Thidé, B., and McCarrick, M.: Unprecedentedly Strong and Narrow Electromagnetic Emissions Stimulated by High-Frequency Radio Waves in the Ionosphere, Phys. Rev. Lett., 102, 065003, doi:10.1103/PhysRevLett.102.065003, 2009.

Porkolab, M.: Theory of parametric instability near the lower-hybrid frequency, Phys. Fluids, 17, 1432-1442, doi:10.1063/1.1694910, 1974

Scales, W. A., Bordikar, M. R., Samimi, A., Bernhardt, P. A., Briczinski, S., Selcher, C. A., and McCarrick, M.: Observations and theory of ion gyro-harmonic structures in the stimulated radiation spectrum during second electron gyro-harmonic heating, General Assembly and Scientific Symposium, URSI, doi:10.1109/URSIGASS.2011.6051126, 2011.

Sharma, R. P., Kumar, A., and Kumar, R.: Excitation of ionBernstein waves in ionospheric modification experiment, Radio Sci., 28, 951-957, doi:10.1029/93RS01374, 1993.

Tereshchenko, E. D., Yurik, R. Yu., Khudukon, B. Z., Rietveld, M. T., Isham, B., Belyey, V., Brekke, A., Hagfors, T., and Grill, M.: Directional features of the downshifted peak observed in HF-induced stimulated electromagnetic emission spectra obtained using an interferometer, Ann. Geophys., 24, 1819-1827, doi:10.5194/angeo-24-1819-2006, 2006. 
Thidé, B., Kopka, H., and Stubbe, P.: Observation of stimulated scattering of a strong high frequency radio wave in the ionosphere, Phys. Rev. Lett., 49, 1561-1564, doi:10.1103/PhysRevLett.49.1561, 1982.
Zhou, H. L., Huang, J., and Kuo, S. P.: Cascading of the upper hybrid/electron Bernstein wave in ionospheric heating experiments, Phys. Plasmas, 1, 3044-3052, doi:10.1063/1.870929, 1994. 